\title{
Association of Serum Homocysteine Levels with Histological Severity of NAFLD
}

\author{
Yilun Xu${ }^{1}$, Yaqi Guan ${ }^{2}$, Xingyi Yang ${ }^{1}$, Ziqiang Xia ${ }^{1}$, Jinming $W^{1}$
}

\author{
1) Department of \\ Gastroenterology, The First \\ Affiliated Hospital of Wenzhou \\ Medical University, Wenzhou \\ 325000, Zhejiang \\ 2) Wenzhou Medical \\ University, Faculty of Nursing, \\ Wenzhou 325000, Zhejiang, \\ China
}

Address for correspondence:
Jinming Wu
Department of
Gastroenterology, The First
Affiliated Hospital of Wenzhou
Medical University
Cai Village on Nanbaixiang
Street,
Ouhai District, Wenzhou,
Zhejiang province,
China
wzfywjm@163.com

Received: 21.10.2019 Accepted: 05.02.2020

\section{ABSTRACT}

Background \& Aims: Studies on the association between homocysteine and non-alcoholic fatty liver disease (NAFLD) have shown inconsistent results. Our study concerns the association of homocysteine with the histological severity of NAFLD, especially non-alcoholic steatohepatitis (NASH) and significant fibrosis (SF) after adjusting for other well-identified risk factors.

Methods: This study enrolled 289 patients with biopsy proven NAFLD. The association of homocysteine with the severe histological features was examined using multivariable logistic regression analysis and subgroup analysis. The area under curves (AUC) and Hosmer-Lemeshow goodness-of-fit test for the adjusted logistic regression models was analyzed.

Results: After multivariable regression analysis, homocysteine showed significant correlation with NASH (OR 0.79 95\%CI: 0.69-0.89), p<0.001) and SF (OR 0.83 95\%CI: 0.72-0.95, p=0.009). Spearman's correlation analysis showed homocysteine levels were inversely correlated with the grade of hepatocellular ballooning and the stage of liver fibrosis (Spearman's $\rho=-0.13, p=0.033$; Spearman's $\rho=-0.16, p=0.007$ ), but had no correlation with the severity of steatosis and lobular inflammation. The subgroup analyses showed that homocysteine was strongly associated with NASH in females but was weaker in males (female OR: 0.61 95\%CI: 0.45-0.84; male 0.86 95\%CI: $0.75-0.99$ ), and on SF showed no significant differences in the subgroups. The models showed good discrimination for NASH (AUC 0.789, 95\% CI: 0.736-0.843) and for SF (0.784 95\%CI: 0.719-0.848) and calibration (Hosmer-Lemeshow goodness-of-fit test, $\mathrm{p}=0.346$ for NASH; $\mathrm{p}=0.908$ for SF).

Conclusion: Elevated serum homocysteine levels are negatively associated with NASH and SF in subjects with NAFLD.

Key words: NAFLD-NASH - significant fibrosis - homocysteine.

Abbreviations: ALP: alkaline phosphatase; ALT: aspartate aminotransferase; AST: alanine aminotransferase; $\gamma$ GT: gamma-glutamyl transferase; HCC: hepatocellular carcinoma; HDL-c: high-density lipoprotein cholesterol; LDL-c: low-density lipoprotein cholesterol; NAFLD: non-alcoholic fatty liver disease; NASH: non- alcoholic steatohepatitis; NAS: NAFLD Active Score; PLT: platelet; ROS: reactive oxygen species; SF: significant fibrosis; TB: total bilirubin; UA: uric acid.

\section{INTRODUCTION}

Non-alcoholic fatty liver disease (NAFLD) is a common cause of chronic liver disease worldwide [1]. The prevalence of NAFLD in the global population is about $25 \%$ and is still rising parallel to western diet, sedentary lifestyle, and increased prevalence of obesity [2]. NAFLD is defined as the presence of steatosis in at least $5 \%$ of hepatocytes, in the exclusion of other liver disease, such as alcoholic liver disease, chronic viral hepatitis, use of medications that induce steatosis and other chronic liver diseases, such as autoimmune hepatitis, Wilson's disease, etc. [3]. It includes a spectrum of liver diseases that ranges from simple fat accumulation in hepatocytes to liver necroinflammation, fibrosis, cirrhosis, and hepatocellular carcinoma (HCC) [4]. Nonalcoholic steatohepatitis (NASH), which is a more progressive type of NAFLD, is characterized by the presence of steatosis, lobular inflammation and hepatocyte ballooning [5]. The patient with steatohepatitis can progress to liver fibrosis more rapidly than without it (7 years per fibrosis stage versus 14 years) [6]. Fibrosis is considered to be the only histological feature that is independently associated with an increased likelihood of liver-related events and all-cause (i.e., cardiovascular disease) 
mortality [7], increasing with the increased stage of fibrosis [8]. Liver biopsy is considered the golden standard for distinguishing NASH from NAFL and staging fibrosis.

In the United States, NAFLD has become the second leading indication for liver transplantation and the third leading cause of HCC, resulting in severe economic burden [9, 10]. Therefore, it is extremely important to fully understand the mechanistic pathways of NAFLD and disease progression. "The multiple-hit" hypothesis that includes insulin resistance, oxidative stress, nutritional factors largely explain the pathogenesis, but knowledge on the mechanisms of NAFLD still remains incomplete [11].

Homocysteine, formed as an intermediary in methionine metabolism, is a sulfur-containing amino acid [12]. It can be remethylated to methionine or catabolized in the transsulfuration pathway to cysteine [13]. The liver is central for the synthesis and catabolism of homocysteine, as it metabolizes the majority of dietary methionine [14]. Thus, it is plausible that changes in homocysteine levels may occur in the event of liver injury. In recent years, a few original studies have explored the association between homocysteine and NAFLD [15-19]. However, the conclusions are still controversial, and the data based on biopsy proven NAFLD are lacking.

For this reason, we studied in biopsy proven NAFLD the posible independent association between serum homocysteine levels and NASH and significant fibrosis (SF) after adjusting for other well-identified risk factors (e.g. metabolic risk factors and insulin resistance).

\section{METHODS}

\section{Patients and study design}

We performed a retrospective, cross-sectional study searching the liver biopsy database from The First Affiliated Hospital of Wenzhou Medical University, Zhejiang, China. The clinical records of 1371 subjects during January 2016 and July 2019 were reviewed. We excluded 1082 subjects because of the following: (1) $\leq 18$-year-old; (2) the steatosis $\leq 5 \%$ of liver cells at histology; (3) excessive alcohol consumption (>140 $\mathrm{g} /$ week for men and $>70 \mathrm{~g} /$ week for women) evaluated by a questionnaire; (4) history of malignancy; (5) history of viral hepatitis, autoimmune hepatitis, or other forms of chronic liver disease; (6) insufficient clinical data. Finally, 289 strictly screened subjects were enrolled in this study. No informed consent was required because all the data were anonymized. The protocol was in accordance with the Helsinki Declaration and was approved by the Ethics Committee of the Medical University.

\section{Data collection and measurements}

Data was collected from the time of liver biopsy. Standing height and body weight of the subjects were measured, when they were barefoot and wearing light clothing. Body mass index (BMI) was categorized as normal: $<24 \mathrm{~kg} / \mathrm{m}^{2}$ and overweight: (based on Chinese criteria): overweight: $\geq 24$. Venous blood sampling was collected after overnight fasting for at least 8-12 hours and measured at the hospital Clinical Sample Test Room. Homocysteine $(\mu \mathrm{mol} / \mathrm{L})$, triglyceride $(\mathrm{TG})(\mathrm{mmol} / \mathrm{L})$, total cholesterol (TC) $(\mathrm{mmol} / \mathrm{L})$, low-density lipoprotein cholesterol (LDL-c) (mmol/L), high-density lipoprotein cholesterol (HDL-c) $(\mathrm{mmol} / \mathrm{L})$, albumin $(\mathrm{g} / \mathrm{L})$, aspartate aminotransferase (ALT) (U/L), alanine aminotransferase (AST) (U/L), alkaline phosphatase (ALP) (U/L), gammaglutamyl transferase $(\gamma \mathrm{GT})(\mathrm{U} / \mathrm{L})$, glucose $(\mathrm{mmol} / \mathrm{L})$, insulin $(\mathrm{mU} / \mathrm{l})$, platelet $(\mathrm{PLT})\left({ }^{\star} 10^{\wedge} 9\right)$, total bilirubin $(\mathrm{TB})(\mu \mathrm{mol} / \mathrm{L})$ and uric acid (UA) $(\mu \mathrm{mol} / \mathrm{L})$ were measured with standard clinical chemistry laboratory techniques. As for the measuring serum homocysteine levels, after about $2 \mathrm{ml}$ venous blood was extracted by the nurse, it was transported to the test room at low temperature and the serum was centrifuged within 1 hour. The serum was stored at $2-8^{\circ} \mathrm{C}$ for testing within 48 hours. The serum homocysteine levels were performed by standard methods using automated techniques (Beckman Coulter AU5811, USA). The test kit was made by Zhejiang Kuake Biotechnology Co., Ltd. Insulin resistance was evaluated using the homeostasis model assessment of insulin resistance (HOMA-IR): fasting blood glucose $(\mathrm{mmol} / \mathrm{l}) *$ insulin $(\mathrm{mU} / \mathrm{l})$ / 22.5 [20]. Diabetes mellitus was diagnosed by fasting blood glucose $\geq 7.0 \mathrm{mmol} / \mathrm{L}$ and/or treatment with antidiabetic drugs. Hypertension was defined as systolic blood pressure $\geq 140 \mathrm{mmHg}$ /diastolic blood pressure $\geq 90 \mathrm{mmHg}$ and/or the current use of anti-hypertensive medication. Smokers were defined as those who had smoked at least one cigarette per day during the previous year.

\section{Liver biopsy}

Liver biopsy was performed by senior operators using $16 \mathrm{G}$ $\mathrm{x} 16 \mathrm{~cm}$ biopsy needles under ultrasonography positioning. The liver specimens were fixed in $10 \%$ formalin and were scored by experienced hepatologists who were blinded to the clinical data, treatment allocation, and imaging findings. A scoring system published by Kleiner et al. [21] was used. The histological NAFLD Active Score (NAS) consists of 0 to 8 points that represent the unweighted sum of the scores for steatosis $(0$ 3), lobular inflammation (0-3), and hepatocellular ballooning $(0-2)$. Subjects with scores of 5 or greater were diagnosed as NASH. Fibrosis was staged as follows: stage $0=$ no fibrosis; stage 1 = perisinusoidal or periportal fibrosis with 3 different patterns: $1 \mathrm{a}=$ mild, zone 3 , perisinusoidal $1 \mathrm{~b}=$ moderate, zone 3 , perisinusoidal fibrosis, and $1 \mathrm{c}=$ portal/periportal fibrosis; stage 2 = perisinusoidal and portal/periportal fibrosis; stage 3 = bridging fibrosis; stage $4=$ cirrhosis. In this study, we pooled the subtype $1 \mathrm{a}, 1 \mathrm{~b}, 1 \mathrm{c}$ of fibrosis into a single F1 score. Significant fibrosis (SF) was defined as stage 2 or greater $(\geq 2)$.

\section{Statistical analysis}

Continuous data were presented as mean \pm standard deviation (normal distribution) or median (1st quartile, 3rd quartile) (skewed distribution), and categorical variables were expressed as frequency or percentage. First, The One-Way ANOVA (normal distribution), Kruskal-Wallis $H$ (skewed distribution) test and chi-square tests (categorical variables) were used to determine any statistical differences between the means and proportions of the groups. Second, the Univariate linear regression model was used to evaluate the associations between variables and NASH and SF, respectively. Third, according to the recommendation of the STROBE statement [22], we simultaneously showed the results from unadjusted, 
minimally adjusted analysis and those from fully adjusted analyses. The covariates, when added to this model, changed the matched odds ratio by at least $10 \%$ and were adjusted. The continuous variables that were entered in the models were all checked for the functional form-the log-linearity assumption. The multicollinearity between variables was checked after multivariable analysis. Fourth, Spearman's correlation analysis was performed to assess the relationship between serum homocysteine levels and histological features. Fifth, subgroup analyses were performed using stratified linear regression models. The modifications and interactions of subgroups were inspected by likelihood ration tests. Lastly, the area under curves (AUC) and Hosmer-Lemeshow goodness-of-fit test for the adjusted logistic regression models was analyzed. All of the analyses were performed with the statistical software packages R (http://www.R-project.org, The R Foundation) and EmpowerStats (http://www.empowerstats.com, X\&Y Solutions, Inc., Boston, MA). A p value less than 0.05 (twosided) was considered statistically significant.

\section{RESULTS}

A total of 289 people were enrolled in this study, with an average age of $41.90 \pm 12.29$ years old, among which $72.66 \%$ were males. Biopsy proven NASH was found in 116 patients (men: 83, 71.6\%). Significant fibrosis was found in 62 subjects (men: $42,67.7 \%$ ). Data were divided into tertiles according to serum homocysteine levels (T1: 7-10, T2: 11-12, T3: 13-36). Baseline characteristics were listed in Table I.

The results of univariate analysis are shown in Table II. BMI, HOMA-IR, serum level of TC, LDL-c, AST, ALT, ALP, $\gamma$ GT, $\mathrm{TB}$ and UA were positively correlated with NASH. Age was negatively associated with NASH. BMI, HOMA-IR and serum level of TC, AST, ALT and ALP were positively correlated with SF.

Multiple regression analysis evaluated the independent associations between homocysteine and NASH, and homocysteine and SF, respectively. Table III shows the nonadjusted and adjusted models. The variable AST did not conform to the linear assumption, so it was not included in the regression analysis. For NASH, in the crude model (not adjusted covariates), homocysteine showed significant correlation with NASH (OR: 0.90, 95\%CI: 0.82-0.98, p=0.018). In the minimally adjusted model I (adjusted for age, BMI), the effect size showed no obvious change (OR: 0.83, 95\%CI: 0.75 0.93, $\mathrm{p}=0.001$ ). After adjusting for model II (adjusted for age, BMI, LDL-c, ALT, $\gamma$ GT, HOMA-IR and UA), these associations were consistently maintained (OR: 0.79, 95\%CI: 0.69-0.89, $\mathrm{p}<0.001)$. As for SF, homocysteine showed no correlation with SF in the crude model (OR: 0.90, 95\%CI: 0.80-1.01, $\mathrm{p}=0.063$ ), but when we adjusted more covariates (model I (adjusted for age, BMI), model II(adjusted for age, BMI, LDL-c, ALT and HOMA-IR)), homocysteine showed a significant correlation with SF (OR: 0.87, 95CI\%: 0.77-0.99, p=0.030; OR: 0.83, 95\%CI: $0.72-0.95, \mathrm{p}=0.009)$. For the purpose of sensitivity analysis, we

Table I. Baseline characteristics of subjects $(\mathrm{n}=289)$

\begin{tabular}{|c|c|c|c|c|}
\hline Homocysteine & $\mathrm{T} 1$ & $\mathrm{~T} 2$ & $\mathrm{~T} 3$ & $\mathrm{p}$ \\
\hline Number & 89 & 91 & 109 & \\
\hline Male, number (\%) & $42(47.19 \%)$ & $71(78.02 \%)$ & $97(88.99 \%)$ & $<0.001$ \\
\hline Diabetes (yes) & $37(41.57 \%)$ & $27(29.67 \%)$ & $22(20.18 \%)$ & 0.005 \\
\hline Hypertension (yes) & $26(29.21 \%)$ & $18(19.78 \%)$ & $19(17.43 \%)$ & 0.116 \\
\hline Smoke (yes) & $9(10.11 \%)$ & $20(21.98 \%)$ & $26(23.85 \%)$ & 0.034 \\
\hline Age (years) & $45(35-54)$ & $42(33-51.5)$ & $38(28-48)$ & 0.003 \\
\hline BMI $\left(\mathrm{kg} / \mathrm{m}^{2}\right)$ & $26.13(24.01-27.83)$ & $26.64(25.22-28.84)$ & $26.63(24.23-29.27)$ & 0.075 \\
\hline $\mathrm{TG}(\mathrm{mmol} / \mathrm{L})$ & $1.67(1.23-2.45)$ & $1.95(1.44-2.62)$ & $1.98(1.31-2.82)$ & 0.165 \\
\hline $\mathrm{TC}(\mathrm{mmol} / \mathrm{L})$ & $5.00(4.14-5.79)$ & $5.30(4.42-5.98)$ & $5.04(4.43-5.83)$ & 0.375 \\
\hline LDL-c (mmol/L) & $2.87(2.28-3.54)$ & $3.05(2.40-3.79)$ & $3.17(2.47-3.65)$ & 0.128 \\
\hline HDL-c (mmol/L) & $1.02(0.88-1.15)$ & $1.02(0.88-1.12)$ & $0.96(0.88-1.09)$ & 0.604 \\
\hline Albumin $(\mathrm{g} / \mathrm{L})$ & $45.4(42.4-47.3)$ & $46.60(44.80-48.95)$ & $47.6(45.4-50)$ & $<0.001$ \\
\hline $\operatorname{ALT}(\mathrm{U} / \mathrm{L})$ & $41(25-88)$ & $59(30-92)$ & $53(33-101)$ & 0.188 \\
\hline $\operatorname{AST}(\mathrm{U} / \mathrm{L})$ & $28(22-54)$ & $36(24.5-55.5)$ & $34(26-58)$ & 0.802 \\
\hline $\operatorname{ALP}(\mathrm{U} / \mathrm{L})$ & $79(63-97)$ & $83(69-96.5)$ & $79(66-94)$ & 0.971 \\
\hline$\gamma \mathrm{GT}(\mathrm{U} / \mathrm{L})$ & $36(25-73)$ & $53(36-85)$ & $55(36-91)$ & 0.135 \\
\hline Glucose $(\mathrm{mmol} / \mathrm{L})$ & $5.70(5.00-6.70)$ & $5.40(4.90-6.75)$ & $5.10(4.70-5.60)$ & 0.003 \\
\hline HOMA-IR & $3.60(1.98-5.06)$ & $3.86(2.59-6.43)$ & $3.88(2.41-5.29)$ & 0.488 \\
\hline $\operatorname{PLT}\left({ }^{*} 10^{\wedge} 9\right)$ & $235(200-293)$ & $224(187-254.5)$ & $243(213-284)$ & 0.016 \\
\hline $\mathrm{TB}(\mu \mathrm{mol} / \mathrm{L})$ & $12(9-16)$ & $13(10-17)$ & $14(11-17)$ & 0121 \\
\hline $\mathrm{UA}(\mu \mathrm{mol} / \mathrm{L})$ & $349(276-393)$ & $384(309-445)$ & $407(349-474)$ & $<0.001$ \\
\hline
\end{tabular}

ALT: aspartate aminotransferase; AST: alanine aminotransferase; ALP: alkaline phosphatase; BMI: Body mass index; $\gamma$ GT: gamma-glutamyl transferase; HDL-c: high-density lipoprotein cholesterol; HOMA-IR: homeostasis model assessment of insulin resistance; LDL-c: low-density lipoprotein cholesterol; PLT: platelet; TB: total bilirubin; TC: total cholesterol; TG: triglyceride; UA: uric acid. 
Table II. The results of univariate analysis

\begin{tabular}{|c|c|c|c|c|c|}
\hline & Statistics & $\begin{array}{l}\text { OR }(95 \% \mathrm{CI}) \text { for } \\
\text { NASH }\end{array}$ & $\mathrm{p}$ & $\begin{array}{l}\text { OR }(95 \% \mathrm{CI}) \\
\text { for SF }\end{array}$ & $\mathrm{p}$ \\
\hline \multicolumn{6}{|l|}{ Sex } \\
\hline Male & $210(72.66 \%)$ & Reference & & Reference & \\
\hline Female & $79(27.34 \%)$ & $1.10(0.65,1.86)$ & 0.728 & $1.36(0.74,2.49)$ & 0.328 \\
\hline \multicolumn{6}{|l|}{ Diabetes } \\
\hline No & $203(70.24 \%)$ & Refrence & & Refrence & \\
\hline Yes & $86(29.76 \%)$ & $0.73(0.43,1.23)$ & 0.236 & $0.87(0.46,1.62)$ & 0.650 \\
\hline \multicolumn{6}{|l|}{ Hypertension } \\
\hline No & $226(78.20 \%)$ & Reference & & Reference & \\
\hline Yes & $63(21.80 \%)$ & $0.63(0.35,1.14)$ & 0.126 & $0.94(0.47,1.87)$ & 0.858 \\
\hline \multicolumn{6}{|l|}{ Smoke } \\
\hline No & $234(80.97 \%)$ & Reference & & Reference & \\
\hline Yes & $55(19.03 \%)$ & $1.09(0.60,1.98)$ & 0.778 & $1.49(0.76,2.93)$ & 0.245 \\
\hline Age (years) & $41.90 \pm 12.29$ & $0.97(0.95,0.99)$ & 0.005 & $1.00(0.98,1.02)$ & 0.907 \\
\hline BMI $\left(\mathrm{kg} / \mathrm{m}^{2}\right)$ & $26.93 \pm 3.46$ & $1.16(1.08,1.24)$ & $<0.001$ & $1.15(1.06,1.24)$ & 0.001 \\
\hline $\mathrm{TG}(\mathrm{mmol} / \mathrm{L})$ & $2.13 \pm 1.11$ & $1.21(0.98,1.50)$ & 0.077 & $1.17(0.92,1.48)$ & 0.206 \\
\hline $\mathrm{TC}(\mathrm{mmol} / \mathrm{L})$ & $5.13 \pm 1.19$ & $1.37(1.11,1.68)$ & 0.003 & $1.31(1.04,1.66)$ & 0.024 \\
\hline LDL-c (mmol/L) & $3.05 \pm 0.97$ & $1.38(1.08,1.77)$ & 0.011 & $1.30(0.98,1.74)$ & 0.069 \\
\hline HDL-c $(\mathrm{mmol} / \mathrm{L})$ & $1.01 \pm 0.20$ & $1.27(0.40,4.09)$ & 0.687 & $1.63(0.41,6.43)$ & 0.487 \\
\hline Albumin (g/L) & $46.30 \pm 3.80$ & $1.05(0.98,1.11)$ & 0.157 & $0.95(0.89,1.03)$ & 0.194 \\
\hline $\operatorname{ALT}(\mathrm{U} / \mathrm{L})$ & $71.03 \pm 60.65$ & $1.02(1.01,1.02)$ & $<0.001$ & $1.01(1.01,1.01)$ & $<0.001$ \\
\hline $\operatorname{AST}(\mathrm{U} / \mathrm{L})$ & $45.25 \pm 33.14$ & $1.04(1.03,1.05)$ & $<0.001$ & $1.02(1.01,1.03)$ & $<0.001$ \\
\hline $\operatorname{ALP}(\mathrm{U} / \mathrm{L})$ & $83.52 \pm 24.00$ & $1.01(1.00,1.02)$ & 0.048 & $1.02(1.00,1.03)$ & 0.009 \\
\hline$\gamma \mathrm{GT}(\mathrm{U} / \mathrm{L})$ & $67.18 \pm 53.58$ & $1.01(1.00,1.01)$ & 0.012 & $1.00(1.00,1.01)$ & 0.215 \\
\hline Glucose $(\mathrm{mmol} / \mathrm{L})$ & $5.76 \pm 1.38$ & $1.00(0.84,1.19)$ & 0.985 & $1.20(0.99,1.44)$ & 0.064 \\
\hline HOMA-IR & $4.98 \pm 4.96$ & $1.15(1.07,1.24)$ & $<0.001$ & $1.13(1.06,1.20)$ & $<0.001$ \\
\hline $\operatorname{PLT}\left({ }^{*} 10^{\wedge} 9\right)$ & $241.49 \pm 61.21$ & $1.00(1.00,1.01)$ & 0.455 & $1.00(1.00,1.01)$ & 0.305 \\
\hline $\mathrm{TB}(\mu \mathrm{mol} / \mathrm{L})$ & $14.63 \pm 7.38$ & $1.04(1.00,1.07)$ & 0.032 & $1.00(0.97,1.04)$ & 0.889 \\
\hline $\mathrm{UA}(\mu \mathrm{mol} / \mathrm{L})$ & $388.62 \pm 108.83$ & $1.00(1.00,1.01)$ & 0.001 & $1.00(1.00,1.00)$ & 0.164 \\
\hline
\end{tabular}

CI: confidence interval; NASH: non-alcoholic steatohepatitis, OR: odds ratio; SF: significant fibrosis; (For abbreviations see Table I).

also handled homocysteine as a categorical variable (tertiles, T1-T3) and found the same results. Using T1 as a reference, the relationship between homocysteine and $\mathrm{NASH}$, homocysteine and SF respectively remained statistically significant in T3 versus T1 (OR: 0.43, 95\%CI: 0.22-0.85, $\mathrm{p}=0.015$; OR: 0.38, 95\%CI: $0.17-0.86, \mathrm{p}=0.020$, respectively) after adjusting for model II, and significant linear trend was observed ( $\mathrm{p}$ for trend $=0.016 ; 0.019$, respectively). Spearman's correlation analysis showed that the serum level of homocysteine was inversely correlated with the grade of hepatocellular ballooning and the stage of liver fibrosis (Spearman's $\rho=-0.13, p=0.033$; $=-0.16, p=0.007)$, but had no correlation with the severity of steatosis and lobular inflammation (Fig. 1).

To determine the effect of potential confounders, the relationship between homocysteine and NASH, homocysteine and SF respectively was further investigated in subgroups (Table IV). The subgroup analysis by gender showed that homocysteine was strongly associated with NASH in females but was weaker in males (female OR: $0.61,95 \% \mathrm{CI}$ : 0.45 to 0.84 ; male OR: $0.86,95 \%$ CI: 0.75 to 0.99 ).
The AUC and Hosmer-Lemeshow goodness-of-fit test for the adjusted logistic regression models was analyzed. For $\mathrm{NASH}$, the model demonstrated good discrimination (the AUC was 0.789, 95CI\%: 0.736 to 0.843 ) (Fig. 2) and calibration (Hosmer-Lemeshow goodness-of-fit test, $\mathrm{p}=0.346$ ). For SF, the model also showed good discrimination (the AUC is 0.784 , 95CI\%: 0.719 to 0.848 ) (Fig. 3) and calibration (HosmerLemeshow goodness-of-fit test, $\mathrm{p}=0.908$ ).

\section{DISCUSSION}

Over the past decade, the relationship between homocysteine and NAFLD has attracted attention, but no unified conclusion has been reached due to different study designs, diagnostic methods of NAFLD, and different sample sizes. To our knowledge, our study is the largest analysis to date that evaluated the association between homocysteine and biopsy proven NAFLD in adults. In our cohort elevated serum homocysteine levels were negatively associated with NASH and SF in subjects with biopsy-proven NAFLD. Homocysteine was 
Table III. Relationship between homocysteine and NASH or SF in different models

\begin{tabular}{|c|c|c|c|c|c|c|}
\hline \multirow[t]{2}{*}{ Variable } & \multicolumn{2}{|c|}{ Crude Model } & \multicolumn{2}{|c|}{ Model I } & \multicolumn{2}{|c|}{ Model II } \\
\hline & OR $(95 \% \mathrm{CI})$ & $\mathrm{p}$ & OR $(95 \% \mathrm{CI})$ & $\mathrm{p}$ & OR $(95 \% \mathrm{CI})$ & $\mathrm{p}$ \\
\hline \multicolumn{7}{|c|}{ Homocysteine as a continuous variable } \\
\hline NASH & $0.90(0.82,0.98)$ & 0,018 & $0.83(0.75,0.93)$ & 0,001 & $0.79(0.69,0.89)$ & $<0.001$ \\
\hline SF & $0.90(0.80,1.01)$ & 0.063 & $0.87(0.77,0.99)$ & 0,030 & $0.83(0.72,0.95)$ & 0.009 \\
\hline \multicolumn{7}{|c|}{ Homocysteine as a categorical variable (tertile) } \\
\hline \multicolumn{7}{|c|}{ NASH } \\
\hline $\mathrm{T} 1(7-10)$ & Reference & & Reference & & Reference & \\
\hline $\mathrm{T} 2(11-12)$ & $0.84(0.46,1.52)$ & 0.562 & $0.66(0.36,1.24)$ & 0.196 & $0.55(0.28,1.08)$ & 0.082 \\
\hline T3 (13-36) & $0.68(0.38,1.21)$ & 0.191 & $0.51(0.28,0.95)$ & 0.034 & $0.43(0.22,0.85)$ & 0.015 \\
\hline Trend test & & 0.190 & & 0.035 & & 0.016 \\
\hline \multicolumn{7}{|l|}{ SF } \\
\hline $\mathrm{T} 1(7-10)$ & Reference & & Reference & & Reference & \\
\hline $\mathrm{T} 2(11-12)$ & $0.91(0.47,1.80)$ & 0.796 & $0.77(0.38,1.55)$ & 0.467 & $0.66(0.31,1.41)$ & 0.285 \\
\hline T3 (13-36) & $0.53(0.26,1.07)$ & 0.077 & $0.47(0.22,0.98)$ & 0.045 & $0.38(0.17,0.86)$ & 0.020 \\
\hline Trend test & & 0.075 & & 0.044 & & 0.019 \\
\hline
\end{tabular}

CI: confidence interval; NASH: non-alcoholic steatohepatitis; OR: odds ratio; SF: significant fibrosis. (For abbreviations see Table I). Crude model did not adjust for other covariates; Model I adjusted for age and BMI: Model II adjusted for age, BMI, LDL-c, ALT, $\gamma$ GT, HOMA-IR and UA to NASH; age, BMI, LDL-c, ALT and HOMA-IR to SF, respectively

strongly associated with NASH in females. In addition, lower homocysteine levels were correlated with progression of liver fibrosis and hepatocellular ballooning [5]. Hepatic steatosis could induce oxidative fat injury, endoplasmic reticulum dysfunction and abnormalities of the cytoskeleton resulting in hepatocellular ballooning. Therefore, hepatocellular ballooning, the hallmark to distinguish steatohepatitis from steatosis with inflammation, is characterized by cellular swelling, rarefaction of the hepatocytic cytoplasm and clumped strands of intermediate filaments [23].

These results were similar a previous study that matched for gender, age and BMI 39 patients with biopsy proven
$\mathrm{P}=\mathbf{0 . 5 4 0}$

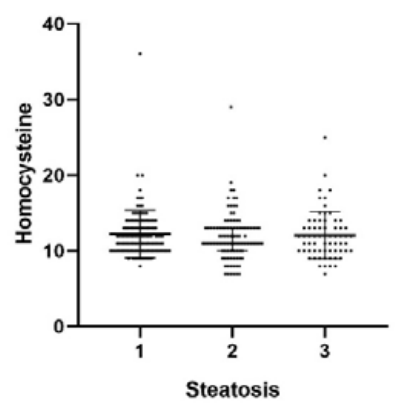

$\mathrm{P}=\mathbf{0 . 2 8 3}$

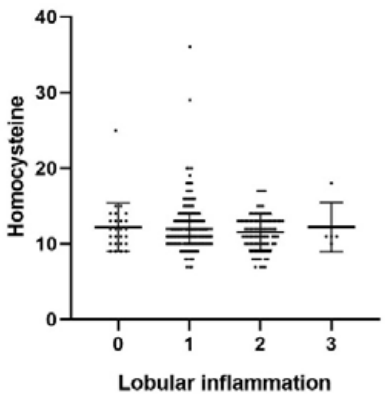

$\mathrm{P}=\mathbf{0 . 0 3 3}$

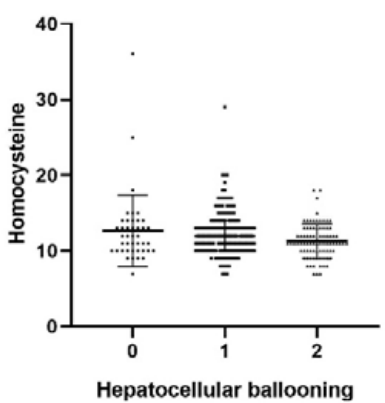

$\mathrm{P}=0.007$

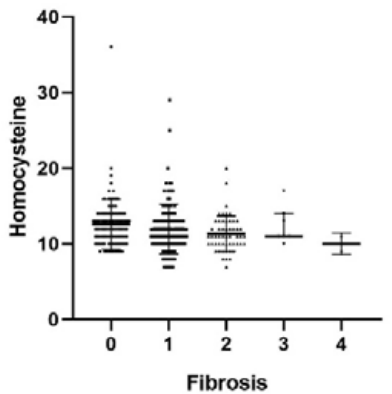

Fig. 1. Correlation analysis between serum homocysteine levels and histological features of NAFLD. The homocysteine showed a strong negative relationship with hepatocellular ballooning and liver fibrosis stage (all $\mathrm{p}<0.05$ ), and no association with steatosis and lobular inflammation. 
Table IV. Effect size of homocysteine on NASH or SF in prespecified and exploratory subgroups

\begin{tabular}{|c|c|c|c|c|c|}
\hline Characteristic & $\mathrm{N}$ & $\begin{array}{c}\text { OR (95\%CI) for } \\
\text { NASH }\end{array}$ & $\begin{array}{c}\mathrm{p} \text { for } \\
\text { interaction }\end{array}$ & $\begin{array}{l}\text { OR }(95 \% \mathrm{CI}) \\
\quad \text { for SF }\end{array}$ & $\begin{array}{c}\mathrm{p} \text { for } \\
\text { interaction }\end{array}$ \\
\hline Gender & & & 0.04 & & 0.78 \\
\hline Male & 210 & $0.86(0.75,0.99)$ & & $0.83(0.69,0.99)$ & \\
\hline Female & 79 & $0.61(0.45,0.84)$ & & $0.87(0.65,1.15)$ & \\
\hline Age (years) & & & 0.60 & & 0.27 \\
\hline$<50$ & 205 & $0.79(0.68,0.91)$ & & $0.86(0.75,1.00)$ & \\
\hline$\geq 50$ & 84 & $0.72(0.53,0.98)$ & & $1.00(0.81,1.22)$ & \\
\hline Smoke & & & 0.99 & & 0.51 \\
\hline Yes & 55 & $0.78(0.58,1.04)$ & & $0.79(0.67,0.93)$ & \\
\hline No & 234 & $0.78(0.68,0.90)$ & & $0.88(0.67,1.14)$ & \\
\hline Hypertension & & & 0.96 & & 0.48 \\
\hline Yes & 63 & $0.77(0.55,1.07)$ & & $0.92(0.66,1.29)$ & \\
\hline No & 226 & $0.78(0.67,0.89)$ & & $0.81(0.68,0.95)$ & \\
\hline Diabetes & & & 0.55 & & 0.67 \\
\hline Yes & 86 & $0.73(0.57,0.95)$ & & $0.86(0.64,1.16)$ & \\
\hline No & 203 & $0.80(0.69,0.92)$ & & $0.80(0.68,0.95)$ & \\
\hline HOMA-IR & & & 0.53 & & 0.59 \\
\hline$<2.5$ & 80 & $0.90(0.66,1.23)$ & & $0.77(0.51,1.15)$ & \\
\hline$\geq 2.5$ & 209 & $0.80(0.70,0.92)$ & & $0.86(0.74,1.00)$ & \\
\hline BMI $\left(\mathrm{kg} / \mathrm{m}^{2}\right)$ & & & 0.34 & & 0.96 \\
\hline$<24$ & 61 & $0.70(0.53,0.94)$ & & $0.87(0.57,1.31)$ & \\
\hline$\geq 24$ & 228 & $0.81(0.71,0.93)$ & & $0.86(0.74,1.00)$ & \\
\hline
\end{tabular}

NAFLD and 22 healthy controls [19]. Inconsistent with our conclusion, two studies in adult Chinese patients found that serum homocysteine level was positively associated with the prevalence of NAFLD, but the diagnosis of hepatic steatosis was made by ultrasonography $[15,16]$. Polyzos et al. [17] concluded that homocysteine was unrelated to NAFLD biopsy proven, but their conclusions were limited by the small sample size (30 patients). Therefore, we hypothesized that the serum homocysteine levels may have a threshold effect on NAFLD. A study in pediatric NAFLD that included 128 age-matched subjects (NAFLD: 64, healthy controls: 64) found similar results with ours; plasma homocysteine levels were significantly increased in NAFLD children as compared to the controls, but significantly decreased in NASH compared to non-NASH

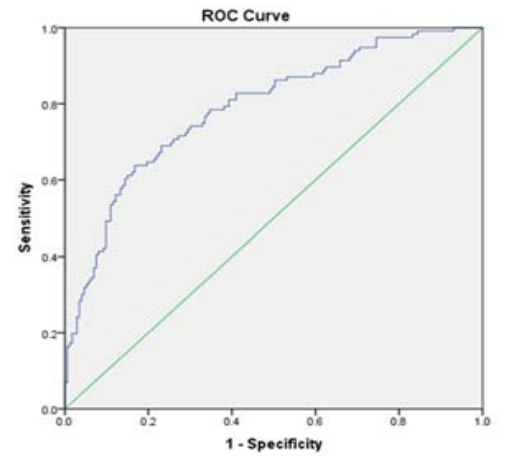

Fig. 2. The area under the curve of the adjusted logistic regression models for NASH. children [18]. However, our study design lacked a healthy control group to test our hypothesis, representing a limit of the present study.

The pathophysiological mechanisms between homocysteine and NAFLD are multifactorial and not fully understood. Deminice et al. [24] suggested that low plasma homocysteine levels may result in decreased glutathione formation leading to increased susceptibility of hepatic cells to reactive oxygen species (ROS). Oxidative stress causes changes in mitochondrial function, depletion of ATP, DNA damage, lipid peroxidation and consequently hepatic inflammation and fibrosis [11,25]. Some studies found significantly lower homocysteine levels in patients with insulin resistance syndrome or high glucose levels compared to control groups [26, 27]. Increased homocysteine

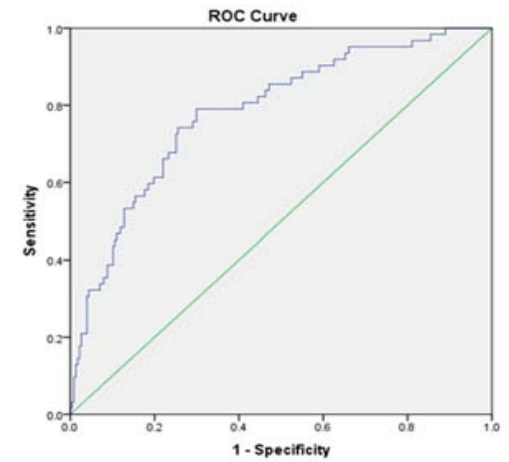

Fig. 3. The area under the curve of the adjusted logistic regression models for SF.. 
levels were associated with weight loss (independent of folate deficiency) in patients after vertical gastroplasty for severe obesity [28]. All of the above-mentioned risk factors, ROS, $\mathrm{BMI}$ and insulin resistance, contribute to the progression of NAFLD [11]. In addition, low levels of homocysteine may lead to decreased production of methionine through remethylating pathway. Methionine is the precursor of S-adenosylmethionine (S-AdoMet), the key methyl donor for phosphatidylcholine synthesis. Low methyl group availability reduces the synthesis of phosphatidylcholine, a major phospholipid required for the assembly and the export of very low-density lipoprotein from the liver, which may lead to hepatic lipid accumulation resulting in hepatic steatosis $[19,29]$. However, some experimental studies have suggested that high plasma homocysteine levels induced hepatocytes in endoplasmic reticulum stress activating both the unfolded protein response and the sterol regulatory element-binding proteins (SREBPs), which causes significant increases in intra-hepatic cholesterol and triglycerides levels leading to the progression of hepatic steatosis [30, 31].

Although our cross-sectional study did not reveal an in-depth mechanism, our finding illustrates the necessity to actively assess serum homocysteine levels in patients with NAFLD. From a clinical point of view, our results suggests that serum homocysteine should be included in the multidisciplinary baseline assessment of patients with NAFLD. Changing the serum levels of homocysteine might be one of the promising potential therapy for NAFLD cure or for preventing progression.

This study had some limitations. Firstly, the cross-sectional design of the study made it possible to investigate associations but not causalities. Secondly, we did not measure folate and vitamin B12 in our study. Finally, this study does not reflect institutional and regional diversities because the cohorts of this study were composed of Chinese patients who were recruited at a single hospital.

\section{CONCLUSION}

Elevated serum homocysteine levels are negatively associated with NASH and SF in subjects with NAFLD. Further prospective cohort studies should establish a causal relationship between homocysteine and NAFLD (NAFL, NASH and SF) and elucidate the associated mechanisms.

Conflicts of interest: None to declare.

Authors' contribution: Y.X., J.W.: study concept; Y.X., Y.G., X.Y., Z.X.: data-collection; Y.X., Y.G.: data analysis and interpretation; Y.X., Y.G., X.Y., Z.X.: drafting of the manuscript; Y.X., J.W.: critical revision of the manuscript for important intellectual content.

\section{REFERENCES}

1. Rinella M, Charlton $M$. The globalization of nonalcoholic fatty liver disease: Prevalence and impact on world health. Hepatology 2016;1:1922. doi:10.1002/hep.28524

2. Younossi ZM, Koenig AB, Abdelatif D, Fazel Y, Henry L, Wymer M. Global epidemiology of nonalcoholic fatty liver disease-Meta-analytic assessment of prevalence, incidence, and outcomes. Hepatology 2016;64:73-84. doi:10.1002/hep.28431

3. Chalasani N, Younossi Z, Lavine JE, et al. The Diagnosis and Management of Non-alcoholic Fatty Liver Disease: Practice Guideline by the American Association for the Study of Liver Diseases, American College of Gastroenterology, and the American Gastroenterological Association. Am J Gastroenterol 2012;6:811-826. doi:10.1038/ ajg.2012.128

4. Satapathy S, Sanyal AJ. Epidemiology and Natural History of Nonalcoholic Fatty Liver Disease. Semin Liver Dis 2015;3:221-235. doi:10.1055/s-0035-1562943

5. Bedossa P. Pathology of non-alcoholic fatty liver disease. Liver Int 2017;37 Suppl 1:85-89. doi:10.1111/liv.13301

6. Singh S, Allen AM, Wang Z, Prokop LJ, Murad MH, Loomba R. Fibrosis Progression in Nonalcoholic Fatty Liver vs Nonalcoholic Steatohepatitis: A Systematic Review and Meta-analysis of Paired-Biopsy Studies. Clin Gastroenterol Hepatol 2015;13:643-654.e1-e9. doi:10.1016/j. cgh.2014.04.014

7. Angulo P, Kleiner DE, Dam-Larsen S, et al. Liver Fibrosis, but No Other Histologic Features, Is Associated With Long-term Outcomes of Patients With Nonalcoholic Fatty Liver Disease. Gastroenterology 2015;149:389-397.e10. doi:10.1053/j.gastro.2015.04.043

8. Dulai PS, Singh S, Patel J, et al. Increased risk of mortality by fibrosis stage in nonalcoholic fatty liver disease: Systematic review and metaanalysis. Hepatology 2017;65:1557-1565. doi:10.1002/hep.29085

9. Wong RJ, Aguilar M, Cheung R, et al. Nonalcoholic Steatohepatitis Is the Second Leading Etiology of Liver Disease Among Adults Awaiting Liver Transplantation in the United States. Gastroenterology 2015;148:547555. doi:10.1053/j.gastro.2014.11.039

10. Younossi ZM, Otgonsuren M, Henry L, et al. Association of nonalcoholic fatty liver disease (NAFLD) with hepatocellular carcinoma (HCC) in the United States from 2004 to 2009. Hepatology 2015;63:1723-1730. doi:10.1002/hep.28123

11. Buzzetti E, Pinzani M, Tsochatzis EA. The multiple-hit pathogenesis of non-alcoholic fatty liver disease (NAFLD). Metabolism 2016;65:10381048. doi:10.1016/j.metabol.2015.12.012

12. Wijekoon EP, Brosnan ME, Brosnan JT. Homocysteine metabolism in diabetes. Biochem Soc Trans 2007;35:1175-1179. Doi:10.1042/ BST0351175

13. FinkelsteinJD. Pathways and regulation of homocysteine metabolism in mammals. Semin Thromb Hemost 2000;26:219-225. doi:10.1055/s-2000-8466

14. Mato JM, Lu SC. Homocysteine, the bad thiol. Hepatology 2005;41:976979. doi:10.1002/hep.20708

15. Dai H, Wang W, Tang X, et al. Association between homocysteine and non-alcoholic fatty liver disease in Chinese adults: a cross-sectional study. Nutr J 2016;15:102. doi:10.1186/s12937-016-0221-6

16. Hu Y, Liu J, Dong X, Xu Y, Leng S, Wang G. Clinical Study of Serum Homocysteine and Non-Alcoholic Fatty Liver Disease in Euglycemic Patients. Med Sci Monitor 2016;22:4146-4151. doi:10.12659/ msm.897924

17. Polyzos SA, Kountouras J, Athanasios AD, Margouta A, Mantzoros CS Association between circulating irisin and homocysteine in patients with nonalcoholic fatty liver disease. Endocrine 2015;49:560-562. doi:10.1007/s12020-014-0473-X

18. Pastore A, Alisi A, di Giovamberardino G, et al. Plasma levels of homocysteine and cysteine increased in pediatric NAFLD and strongly correlated with severity of liver damage. Int J Mol Sci 2014;15:21202 21214. doi:10.3390/ijms151121202 
19. Polyzos SA, Kountouras J, Patsiaoura K, et al. Serum homocysteine levels in patients with nonalcoholic fatty liver disease. Ann Hepatol 2012;11:68-76. doi:10.1016/S1665-2681(19)31488-7

20. Matthews DR, Hosker JP, Rudenski AS, Naylor BA, Treacher DF, Turner RC. Homeostasis model assessment: insulin resistance and beta-cell function from fasting plasma glucose and insulin concentrations in man. Diabetologia 1985;28:412-419. doi:10.1007/bf00280883

21. Kleiner DE, Brunt EM, Van Natta M, et al. Design and validation of a histological scoring system for nonalcoholic fatty liver disease. Hepatology 2005;41:1313-1321. doi:10.1002/hep.20701

22. Vandenbroucke JP, Von Elm E, Altman DG, et al. Strengthening the Reporting of Observational Studies in Epidemiology (STROBE): explanation and elaboration. Ann Intern Med 2007;147:W163-W194. doi:10.7326/0003-4819-147-8-200710160-00010-w1

23. Caldwell S, Ikura Y, Dias D, et al. Hepatocellular ballooning in NASH J Hepatol. 2010;53:719-723. doi:10.1016/j.jhep.2010.04.031

24. Deminice R, Portari GV, Marchini JS, Vannucchi H, Jordao AA. Effects of a low-protein diet on plasma amino acid and homocysteine levels and oxidative status in rats. Ann Nutr Metab 2009;54:202-207. doi: $10.1159 / 000218707$

25. Rolo AP, Teodoro JS, Palmeira CM. Role of oxidative stress in the pathogenesis of nonalcoholic steatohepatitis. Free Radic Bio Med 2012;52:59-69. doi:10.1016/j.freeradbiomed.2011.10.003
26. Bar-On H, Kidron M, Friedlander Y, et al. Plasma total homocysteine levels in subjects with hyperinsulinemia. J Intern Med 2000;247:287294. doi:10.1046/j.1365-2796.2000.00592.x

27. Rosolová H, Simon J, Mayer Jr O, Racek J, Dierzé T, Jacobsen DW. Unexpected inverse relationship between insulin resistance and serum homocysteine in healthy subjects. Physiol Res 2002;51:9398.

28. Borson-Chazot F, Harthe C, Teboul F, et al. Occurrence of hyperhomocysteinemia 1 year after gastroplasty for severe obesity. J Clin Endocrinol Metab 1999;84:541-545. doi:10.1210/ jcem.84.2.5476

29. Obeid R, Herrmann W. Homocysteine and lipids: S-Adenosyl methionine as a key intermediate. FEBS Lett 2009;583:1215-1225. doi:10.1016/j.febslet.2009.03.038

30. Aissa AF, Tryndyak V, de Conti A, et al. Effect of methionine-deficient and methionine-supplemented diets on the hepatic one-carbon and lipid metabolism in mice. Mol Nutr Food Res 2014;58:1502-1512. doi:10.1002/mnfr.201300726

31. Werstuck GH, Lentz SR, Dayal S, et al. Homocysteine-induced endoplasmic reticulum stress causes dysregulation of the cholesterol and triglyceride biosynthetic pathways. J Clin Invest 2001;107:1263-1273. doi:10.1172/JCI11596 